\title{
Protective Effect of Aloe vera (Aloe barbadensis Miller) on Erythrocytes Anion Transporter and Oxidative Change
}

\author{
Sergio Mazzulla ${ }^{1}$, Settimio Sesti ${ }^{1}$, Anita Schella ${ }^{1}$, Ida Perrotta ${ }^{2}$, Adelaide Anile $^{3}$, Saverio Drogo ${ }^{4}$ \\ ${ }^{1}$ Department of Cell Biology, University of Calabria, Rende, Italy; ${ }^{2}$ Department of Ecology, University of Calabria, Rende, Italy; \\ ${ }^{3}$ Research Area MASDER sas, Marano Marchesato, Italy; ${ }^{4}$ Drogo Farm, Rocca Imperiale, Italy. \\ Email: mazzulla@unical.it
}

Received October $16^{\text {th }}, 2012$; revised November $16^{\text {th }}$, 2012; accepted November $24^{\text {th }}, 2012$

\begin{abstract}
The purpose of this study was to evaluate the ability of aqueous extract of Aloe barbadensis Miller (Aloe vera) on oxidative damage and Anion Exchanger 1 (AE1, also known as Band 3) expression in human erythrocytes exposed to the water soluble free radical initiator 2.2'-azobis-2-amidinopropano dihydrochloride (AAPH). In addition, total phenolic compounds in the extracts were determined as catechin equivalent and the various antioxidant activities were compared to natural and synthetic standard antioxidants such as BHA and ascorbic acid. Since Aloe vera extract did not cause a consumption of the cytosolic antioxidant, glutathione (GSH) when it was direct incubated with GSH in basic aerated aqueous solution, this indicates that Aloe vera extract does not proceed auto oxidation at this experimental condition. Furthermore, Aloe vera extract prevent the consumption of GSH, in radical treated RBCs. It also inhibit consumption of GSH when it was direct incubated with AAPH. Aloe vera gel extract inhibits the generation of diphenyl-2-picrylhydrazyl (DPPH) and the scavenging activity was increased in a dose dependent manner. Aloe vera extract was shown the similar reducing power than standards BHT and ascorbic acid. Biochemical analysis by SDS-PAGE and western blotting showed that AAPH-induced oxidative stress increased the susceptibility of AE1 to proteolytic degradation. Of note, our data evidenced that Aloe vera treatment was able to partially restore the normal RBC membrane protein profiles in a dose-dependent manner. These results clearly demonstrate the antioxidative activity of Aloe vera gel extract that might be ascribed to a synergistic action of the bioactive compounds contained therein.
\end{abstract}

Keywords: AE1 Transporter; In Vitro Oxidative Damage; Aloe barbadensis Miller; Antioxidant; Red Blood Cells Membrane Protein; Free Radicals

\section{Introduction}

Aloe vera (L.) BURM. fil. (synonym A. barbadensis MILLER) (Liliaceae), is a perennial succulent plant belonging to the Aloeaceae family, a sub-family of the Asphodelaceae [1]. The plant is composed of turgid green leaves joined at the stem in a rosette pattern. Each leaf consists of an outer green rind (skin) and an inner clear pulp (gel) contained water soluble and fat-soluble vitamins, minerals, enzymes, polysaccharides, phenolic compounds and organic acids [2]. Studies have reported that Aloe vera gel has many activity including anti-inflammatory [3], antibacterial, antioxidant [4], hypoglycemic and hypolipidemic properties [5,6], and also exert diverse pharmacological and therapeutic activities [7]. As source of synergistic bioactive compounds, it includes anthraquinones, glycoproteins, polysaccharides, vitamins and enzymes [8]. In addition several works reported on the effect of consumption of Aloe vera gel on gastric ulcer, gastric microcirculatory changes, hepatoprotective and clinical treatment of sepsis $[9,10]$. The Aloe vera gel could be useful to neutralize free radicals for maintaining the integrity of the antioxidant status. According to this potential activity, the aim of this work was to study the properties of Aloe vera as radical scavenger protecting Red Blood Cell Membrane (RBCM) from 2,2,-Azobis (2-methylpropionamide) dihydrochloride (AAPH) induced oxidative injury and preserving the RBC Anion Exchanger 1 (AE1), also called Band 3. This transporter is $95 \mathrm{kDa}$ protein is composed of two functionally distinct domains. The $43 \mathrm{Kda} \mathrm{N}$-terminal cytoplasmic domain of band 3 provides an anchor for the membrane skeleton via interaction with ankyrin, protein 4.1 , and protein 4.2. The $53 \mathrm{kDa}$ C-terminal membrane domain that is thought to traverse the bilayer 12 times and exchanges $\mathrm{Cl}^{-}$for $\mathrm{HCO}_{3}^{-}$across the plasma membrane during the gas transporter in the blood and also mediates removal of senescent RBCs from circulation. Since quan- 
titative changes in this protein are important in maintaining cell morphology [11], we evaluated the RBCM modification as manifested by a decreasing cytoskeletal protein content, which can lead to abnormalities in RBC shape and disturbances in the microcirculation $[12,13]$.

\section{Matherial and Methods}

\subsection{Chemicals and Matherials}

The following chemicals were used: Diphenyl-1-picrylhydrazyl (DPPH), Potassium phosphate, Catechin hydrate, Butylated hydroxytoluene (BHT), Sodium phosphate dibasic, Sodium phosphate monobasic, Potassium ferricyanide(III), Trichloroacetic acid (TCA), Iron(III) chloride, L-Ascorbic Acid, L-Glutathione reduced (GSH), Sodium tetraborate decahydrate, Boric Acid, 5,5'-Dithiobis (2-nitrobenzoic acid) (DTNB), Phosphate buffered saline (PBS), Methanol, Sodium carbonate, meta-Phosphoric acid, Ethylenediaminetetraacetic acid (EDTA), Phenylmethanesulfonyl fluoride (PMSF), Acrylamide, N,N'-Methylenebis (acrylamide), (hydroxymethyl) aminomethane (Tris), Sodium dodecyl sulfate (SDS), Ammonium persulfate (APS), N,N,N',N'-Tetramethylethylenediamine (TEMED), Glycine, Red Ponceau, Bovin Serum Albumins (BSA), Tween 20, Whatman ${ }^{\circledR} 3 \mathrm{MM}$ paper, Protran $^{\circledR}$ nitrocellulose membranes $0,45 \mu \mathrm{m}$, were purchased from Sigma Chemical Co. (St.Lous, MO). Potassium ferricyanide, Ferric chloride, Sodium Chloride, and Folin-Ciocalteu phenol reagent were acquired from Merck (Milan, Italy). Mouse monoclonal anti-Band 3 and anti$\beta$-actin antibodies were obtained from Santa Cruz Biotechnology (Heidelberg, Germany). Biotinylated antihorse IgG and Avidin-biotin HRP visualization systems (Vecstain ${ }^{\circledR}$ ABC kit PK-6100) were procured Vector Laboratories, Inc (California, USA). 7315 UV/V spectrophotometer (Jenway, Staffordshire, United Kingdom) was used for spectrophotometric assay. Centrifuge centric 200/R (Techtnica, Železniki, Slovenia) was used for centrifugations.

\subsection{Plant Material}

Phenolic substances, known to be responsible for the antioxidant activity of plant extracts, are mostly extracted by organic solvents but as plants are commonly consumed as water extracts by people, the aqueous extract of Aloe vera leaves were preferably used in our investigation. Mature Aloe vera leaves was collected from Drogo farm, Rocca Imperiale, Cosenza (Italy).

\subsection{Aloe vera Leaf Gel Extract}

The gel (100 $\mathrm{g}=0.75 \mathrm{~g}$ dry matter) was homogenized in a electric blender, then diluted with an equal volume of
PBS and homogenized for a second time. The extract was kept at $4^{\circ} \mathrm{C}$ overnight, then filtered through cloth. The clear filtrate was kept at $-20^{\circ} \mathrm{C}$ in aliquots.

\subsection{Total Phenols Content (TPC)}

Aliquot of $0.1 \mathrm{ml}$ of Aloe vera gel extract was made up to $4.6 \mathrm{ml}$ with distilled water in a tube. After addition of 0.1 $\mathrm{ml}$ Folin-Ciocalteu reagent (previously diluted 3-fold with distilled water) and $0.3 \mathrm{ml} 2 \%$ aqueous sodium carbonate solution, tubes were vortexed and the absorbance of the blue color that developed in each assay mixture was recorded after $2 \mathrm{~h}$ at $760 \mathrm{~nm}$, against a blank containing 0.1 $\mathrm{ml}$ of the extraction solvent [14]. Catechin hydrate (0.1 $0.8 \mathrm{mg} / \mathrm{g}$ ) was used for calibration of a standard curve. The results were expressed as grams Catechin Equivalents (CEs)/L of gel extract. The data were presented as the average of triplicate analyses.

\subsection{DPPH Free Radical Scavenging}

Free radical scavenging activity was determined using DPPH assay [15]. When DPPH reacts with an antioxidant compound which can donate hydrogen, it is reduced. The change in color from deep violet to golden/light yellow can be measured at $517 \mathrm{~nm}$. Briefly, $1 \mathrm{~mL}$ of $0.3 \mathrm{mM}$ of DPPH solution was added to $1 \mathrm{~mL}$ of Aloe vera gel extract (5 - $30 \mu \mathrm{M} \mathrm{CEs),} \mathrm{and} \mathrm{incubated} \mathrm{in} \mathrm{the} \mathrm{dark} \mathrm{at}$ room temperature for $30 \mathrm{~min}$. Inhibition of free radical DPPH in percent (I\%) was calculated from the absorption according to the following equation:

$$
\begin{aligned}
& \text { Scavenging effect }[\%] \\
& =\left(\frac{1-\text { Abs sample } 517 \mathrm{~nm}}{\text { Abs control } 517}\right) \times 100
\end{aligned}
$$

Ten independent experiments were carried out and results were expressed as mean values \pm standard error of the mean (S.E.M.). The extract concentration providing $50 \%$ inhibition (EC50\%) was calculated from the graph of scavenging effect percentage against the extract concentration. Ascorbic acid and BHT were used as standards.

\subsection{Reducing Power}

Aloe vera gel extract (5 - $30 \mu \mathrm{M}$ CEs) were mixed with $200 \mathrm{mM}$ PBS (pH 6.6) and $1 \% \mathrm{~K}_{4}\left[\mathrm{Fe}(\mathrm{CN})_{6}\right]$. The mixture was incubated at $50^{\circ} \mathrm{C}$ for $20 \mathrm{~min}$ and $10 \%$ TCA was then added to the mixture and centrifuged $650 \mathrm{~g}$ for 10 $\mathrm{min}$. The upper layer of solution was mixed with distilled water and $0.1 \% \mathrm{FeCl}_{3}$. The increase in absorbance at 700 $\mathrm{nm}$ of the reaction mixture indicated reducing power [16]. BHT and Ascorbic Acid were used as standards. 


\subsection{Autooxidation}

Aloe vera gel (50 - $500 \mu \mathrm{M}$ CEs) was incubated with GSH $(100 \mu \mathrm{M})$ in a borate buffer (50 mM, pH 9.25). An appropriate volume of the reaction mixture was removed at intervals and added to an aqueous solution of DTNB (0.2 mM, pH 9.0). After 3 min incubation, absorbance at $412 \mathrm{~nm}$ was measured and thio concentrations calculated by using [17]:

$$
\varepsilon 412 \mathrm{~nm} \times 13,600 \mathrm{~cm}^{-1} \cdot \mathrm{M}^{-1}
$$

\subsection{Preparation of RBCs Suspension}

Blood was obtained from donor volunteers (10 men aged $47 \pm 11$ years) by venipuncture, and collected into tubes containing ethylenediaminetetraacetic acid (EDTA) as an anticoagulant. The samples were centrifuged at $1100 \mathrm{~g} /$ min for 10 minutes; plasma and buffy coat were carefully removed and discarded. RBCs were washed three times with PBS pH 7.4. RBCs used on the day were preincubated with AAPH at final concentration of $50 \mathrm{mM}$. In all sets of experiments incubations of RBCs were carried out at $37^{\circ} \mathrm{C}$ for 180 minutes in the presence of AAPH with and without Aloe vera gel under gentle shaking. Untreated RBCs has been used as control.

\subsection{Glutathione Content in RBCs}

Blood GSH level was measured spectrophotometrically using Ellmans reagent (DTNB) as a coloring reagent as the method described by Beutler et al. [18]. Briefly, 0.2 $\mathrm{ml}$ blood was mixed with $1.8 \mathrm{ml}$ double distilled water and glacial meta-phosphoric acid (a precipitating solution) was added, centrifuged and supernatant was collected. Lastly, the supernatant was mixed with $300 \mathrm{mM}$ disodium hydrogen sulphate and DTNB reagent and was allowed to stand for 2 min at room temperature. The absorbance was read at $412 \mathrm{~nm}$. GSH values were determined using the molar extinction coefficient of 13,600 $\mathrm{cm}^{-1} \cdot \mathrm{M}^{-1}$.

\subsection{Preparation of RBCs Membrane}

Performed RBCs were lysed in 20 volumes of lysis buffer (5 mM sodium phosphate, $1 \mathrm{mM}$ EDTA, $\mathrm{pH}$ 7.9), in presence of the protease inhibitor phenylmethylsulfonyl fluoride (final concentration of $0.1 \mathrm{mM}$ ) were centrifuged (20 min at $4^{\circ} \mathrm{C}, 13500 \mathrm{~g}$ ). To removal from the button granulocyte debris, the membrane pellet was re-suspended in the above buffer and centrifuged once more. The ghosts were washed 5 - 7 times in a similar manner until white [19]. The protein concentration of the RBC membrane suspensions was determined by Bradford method [20].

\subsection{Western Blotting}

Lysates (25 $\mu \mathrm{g}$ ) were equal loaded for 5 min with $\beta$-actin and then subjected to $10 \%$ SDS-PAGE and transferred to nitrocellulose. The membrane was blocked in low-fat (1\%) milk diluted 1:20 into TBST (10 mM Tris $\mathrm{HCl}[\mathrm{pH}$ 7.5], $50 \mathrm{mM} \mathrm{NaCl}, 0.1 \%$ Triton X-100) for $30 \mathrm{~min}$, followed by incubation for $1.5 \mathrm{~h}$ with the anti-Band 3 antibody. Membranes then were washed extensively with TBST and immuneoreactive protein was detected by incubation with biotinylated anti-horse IgG. Protein was visualized using Vecstain ${ }^{\circledR}$ ABC kit.

\subsection{Statistical Analysis}

Statistical analyses of the results were performed using one and two-way ANOVA followed by Bonferroni's test. $\mathrm{P}$ value $<0.05$ was considered statistically significant.

\section{Results}

\subsection{Total Phenols Content}

The antioxidant activity of plant materials closely correlated with the content of their phenolic compounds [21]. The Aloe vera gel extract was shown to contain $1.34 \pm$ 0.087 grams of $\mathrm{CEs} / \mathrm{L}$.

\subsection{Reducing Power}

The presence of reductants in the samples tested would result in the reducing of $\mathrm{Fe}^{3+}$ to $\mathrm{Fe}^{2+}$ by donating an electron. Amount of $\mathrm{Fe}^{2+}$ complex can then be monitored by measuring the formation of Perl's blue at $700 \mathrm{~nm}$. Figure 1 shows the reducing power of Aloe vera gel and the reference compounds, BHT and Ascorbic Acid. Here, Aloe Vera gel (10 $\mu \mathrm{M}$ CEs) was shown the same reducing power than standards.

\subsection{DPPH Radical Scavenging Activity}

Aloe vera gel extract inhibits the generation of DPPH radical in a dose dependent manner and its $\mathrm{IC}_{50}$ value was found to be $2.9 \pm 0.1 \mu \mathrm{g} / \mathrm{ml}(10 \mu \mathrm{M}$ CEs $)$, which is defined as the concentration of substrate that causes $50 \%$ loss of the DPPH activity (color). A lower value of $\mathrm{IC}_{50}$ indicates the greater antioxidant activity of a test substance. Figure 2 shows the radical scavenging ability of Aloe vera gel and the reference compounds.

\subsection{Autooxidation of $\boldsymbol{A}$. vera Gel Extract}

AAPH induce a decrease in sulfhydryl groups after incubation with glutathione in basic aerated aqueous solution (borate buffer, $\mathrm{pH}$ 9.25). Aloe vera gel extract did not cause a consumption of GSH when it was direct incubation with AAPH (Figure 3). 


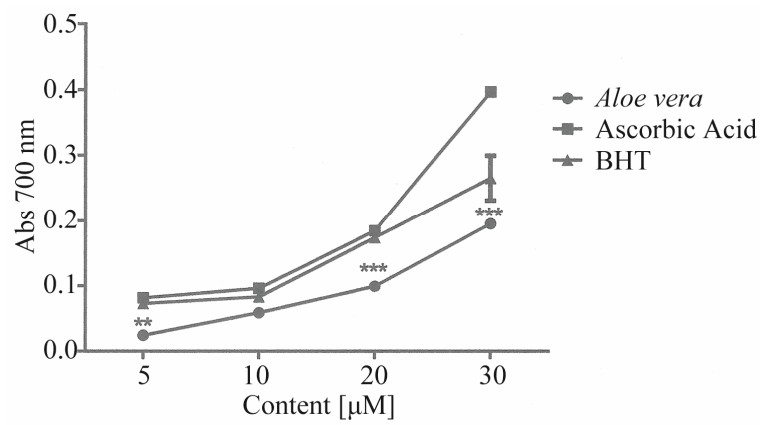

Figure 1. Reducing power of Aloe vera gel vs BHT and ascorbic acid. Values are mean \pm S.E.M. of ten independent determinations. Two-way ANOVA, significant different from ascorbic acid and BHT: “**” $\mathbf{P}<0.01$; “***” $\mathbf{p}<$ 0.001 .

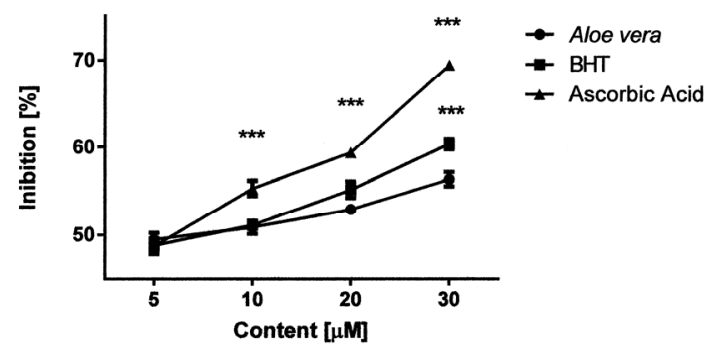

Figure 2. Antioxidant ability of Aloe vera gel extract vs BHT and ascorbic acid. Value are mean \pm S.E.M. of ten independent determinations. Two-way ANOVA, significant different from $A$. Vera gel: “***” $\mathrm{p}<0.001$.

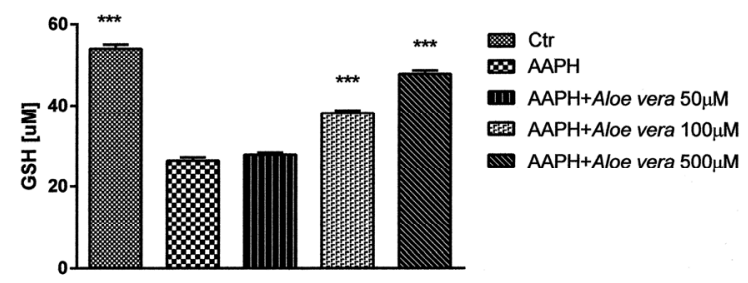

Figure 3. Autooxidation of Aloe vera gel extract and consumption of GSH by AAPH. Value are mean \pm S.E.M. of ten independent determinations. One-way ANOVA, significant different from AAPH “***» $\mathrm{p}<0.001$.

\subsection{Effects of $\boldsymbol{A}$. vera Gel Extract on GSH Content}

The GSH content of untreated RBCs at $37^{\circ} \mathrm{C}$ after 180 min was $0.37 \pm 0.029 \mu \mathrm{M}$. AAPH $(50 \mathrm{mM})$ induced a rapid consumption of cytosolic GSH $(0.25 \pm 0.015 \mu \mathrm{M})$. Addition of different concentrations Aloe vera gel extract (5, 10, 20 and $30 \mu \mathrm{M} \mathrm{CEs}$ ), prevented the GSH consumption induced by AAPH, (Figure 4).

\subsection{Effects of $A$. vera Gel Extract on AAPH-Induced Changes in RBCM Proteins}

Western Blot analysis of RBCM isolated after AAPH in-

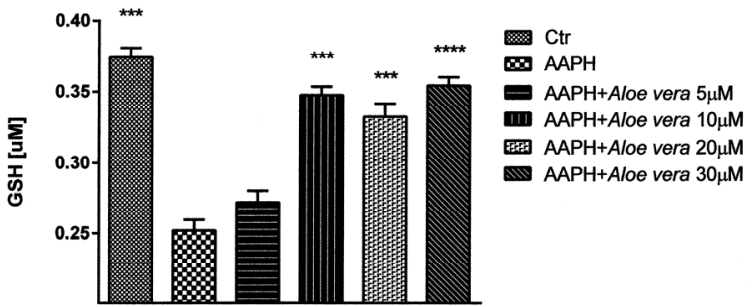

Figure 4. Effects of Aloe vera gel extract on AAPH-induced depletion of intracellular GSH content in RBCs. Values are mean \pm S.E.M. of ten independent determinations. One-way ANOVA, significant different from AAPH “***» $\mathbf{p}<\mathbf{0 . 0 0 1}$.

cubation with and without Aloe vera gel extract (5 - 30 $\mu \mathrm{M}$ CEs) were performed with mouse monoclonal antibody anti-Band 3 (Figure 5). Our dates showed: (a) an increased susceptibility to proteolysis breakdown of AE1 transporter during oxidative damage (lane 2) compared with the intact Band 3 in untreated, control sample (lane 1); (b) a reduction of Band-3 degradation after incubation with Aloe vera gel extract in a dose dependent manner (lanes 3-6); $\beta$-actin was used as loading control.

\section{Discussion}

DPPH scavenging activity showed the effectiveness of the plant extract in donating hydrogen proton to the lone pair electron of the radical. Since Aloe vera gel extract inhibits the generation of DPPH radical in a dose dependent manner, it could be assumed that gel extract contains compounds capable of donating protons to the free radicals thus demonstrating its reducing power. This property is generally associated with the presence of reductones which have been reported react with certain precursors of peroxide, thus preventing their formation [22]. Much of the evidence on the beneficial effects of dietary polyphenols is derived from experiments performed in vitro or in animal models, and by using concentrations much higher than those generally contained in the human diet. For instance, the range of concentrations required for an effect in vitro varies from $0.1 \mu \mathrm{M}$ to $100 \mu \mathrm{M}$ while physiologic concentrations do not exceed $10 \mu \mathrm{M}$ [23]. In accordance with these previous results, our data demonstrate that Aloe vera extract (and its bioactive compounds) at a concentration of $5-10 \mu \mathrm{M}$ of catechin equivalent is capable to prevent consumption of GSH and the protein breakdown induced by AAPH at the experimental condition. Radical attack could be the result of GSH oxidation that occur indirectly through the reduction of reactive thiol groups in oxidized membrane proteins. Antiradical properties of Aloe vera gel extract could be implicated in the regeneration of GSSG in its active reduced form. These results could explain why Aloe vera gel extract did not proceed AAPH-induced depletion of intracellular 


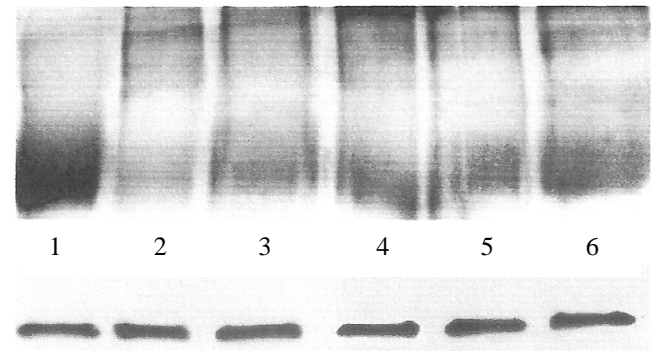

Figure 5. Western blotting analysis of RBCM prepared from the reaction mixture. Lane 1 , intact $R B C$ membrane proteins from control sample. Lane 2, RBC exposed to AAPH (50 mM) at $37^{\circ} \mathrm{C} C$ for $3 \mathrm{~h}$. Lane 3 - 6, RBC exposed to $\mathrm{AAPH}$ in the presence of 5, 10, 20 and $30 \mu \mathrm{M}$ Aloe vera gel extract. The amount of protein loaded was $25 \mu \mathrm{g}$ in each case, $\beta$-actin serves as a loading control.

GSH content in RBCs. It is well known that AAPH can exert formation of High Molecular Weight (HMW) proteins with a concomitant decrease of Low Molecular Weight (LMW) proteins [24]. In accordance, our Western Blotting analysis showed that Aloe vera gel extract inhibited AAPH-induced changes in RBC membrane proteins in a concentration dependent manner. AAPH-induced proteolysis of AE1 is thought to play a pivotal role in the assembly of the membrane skeleton which is crucial for the mechanical integrity of RBCs [25,26]. A loss of interaction between band 3 and the cytoskeleton may be responsible, at least in part, for an increased susceptibility of band-3 to crosslinking which, in turn, could lead to membrane vesiculation. Vesicle release in vivo is considered an integral part of the physiological aging process, enabling the removal of damaged membrane patches from otherwise functional erythrocytes [26].

\section{Conclusion}

The present study demonstrates that Aloe Vera gel extract induces beneficial effects in terms of protection against reduction of GSH content, RBCM protein breakdown and band-3 degradation caused by AAPH. Our results also underline the important role of Aloe vera gel extract in the maintenance of the antioxidant status and antioxidant defense in RBCs. This scavenging activity might be due to the synergistic actions of bioactive compounds present in the plant extracts. We suggest that dietary Aloe vera supplementation can help to prevent oxidative stress and might be also useful for the treatment of oxidative stress-related human disorders by virtue of its antioxidant activity.

\section{REFERENCES}

[1] U. Eggli, "Illustrated Handbook of Succulent plants: Monocotyledons,” Springer, New York, 2001.

\section{doi:10.1007/978-3-642-56715-5}

[2] M. D. Boudreau and F. A. Beland, "An Evaluation of the Biological and Toxicological Properties of Aloe Barbadensis (Miller), Aloe Vera," Journal of Environmental Science and Health Part C Environmental Carcinogenesis \& Ecotoxicology Reviews, Vol. 24, No 1, 2006, pp. 103-154. doi:10.1080/10590500600614303

[3] P. Rishi, A. Rampuria, R. Tewari and A. Koul, "Phytomodulatory Potentials of Aloe vera against Salmonella OmpR-Mediated Inflammation,” Phytotherapy Research, Vol. 22, No. 8, 2008, pp.1075-1082. doi/10.1002/ptr.2458/abstract

[4] O. A. Wintola and A. J. Afolayan, "Phytochemical Constituents and Antioxidant Activities of the Whole Leaf Extract of Aloe Ferox Mill,” Pharmacognosy Magazine, Vol. 7, No. 28, 2011, pp. 325-333. doi:10.4103/0973-1296.90414

[5] F. Habeeb, E. Shakir, F. Bradbury, P. Cameron, M. R. Taravati, A. J. Drummond, A. I. Gray and V. A. Ferro, "Screening Methods Used to Determine the Anti-Microbial Properties of Aloe vera Inner Gel,” Methods, Vol. 42, No. 4, 2007, pp. 315-320. doi:10.1016/j.ymeth.2007.03.004

[6] K. Kim, H. Kim, J. Kwon, S. Lee, H. Kong, S.-A. Im, Y.-H. Lee, Y.-R. Lee, S.-T. Oh, T. H. Jo, Y. l. Park and C.-K. Lee, "Hypoglycemic and Hypolipidemic Effects of Processed Aloe vera Gel in a Mouse Model of Non-Insulin-Dependent Diabetes Mellitus,” Phytomedicine, Vol. 16, No. 9, 2009, pp. 856-863. doi.org/10.1016/j.phymed.2009.02.014

[7] V. Steenkamp and M. J. Stewart, "Medicinal Applications and Toxicological Activities of Aloe Products," Pharmaceutical Biology, Vol. 45, No. 5, 2007, pp. 411-420. doi:10.1080/13880200701215307

[8] J. H. Hamman, "Composition and Applications of Aloe vera Leaf Gel,” Molecules, Vol. 13, No 8, 2008, pp. 1599-1616.

[9] M. A. Gbadegesin, O. A. Odunola, K. A. Akinwumi and O. O. Osifeso, "Comparative Hepatotoxicity and Clastogenicity of Sodium Arsenite and Three Petroleum Products in Experimental Swiss Albino Mice: The Modulatory Effects of Aloe vera Gel," Food and Chemical Toxicology, Vol. 47, No. 10, 2009, pp. 2454-2457. doi:10.1016/j.fct.2009.07.002

[10] N. Yun, C. H. Lee and S. M. Lee, "Protective Effect of Aloe vera on Polymicrobial Sepsis in Mice," Food and Chemical Toxicology, Vol. 47, No. 6, 2009, pp. 13411348. doi:10.1016/j.fct.2009.03.013

[11] M. J. A. Tanner, "The Structure and Function of Band 3 (AE1): Recent Developments (Review),” Molecular Membrane Biology, Vol.14, No. 4, 1997, pp. 155-165. doi:10.3109/09687689709048178

[12] L. J. Bruce and M. J. A. Tanner, "Erythroid Band 3 Variants and Disease," Baillière's Clin Haematol, Vol. 12, No. 4, 1999, pp. 637-654. doi:10.1053/beha.1999.0046

[13] T. Somer and H. J. Meiselman, "Disorders of Blood Viscosity,” Annals of Medicine, Vol. 25, No. 1, 1993, pp. 31-39. doi:10.3109/07853899309147854 
[14] K. Slinkard and V. L. Singleton, "Total Phenol Analyses: Automation and Comparison with Manual Methods," American Journal of Enology and Viticulture, Vol. 28, No. 1, 1977, pp. 49-55.

[15] W. Brand-Williams, M. E. Cuvelier and C. Berset, "Use of a Free Radical Method to Evaluate Antioxidant Activity," LWT-Food Science and Technology, Vol. 28, No. 1, 1995, pp. 25-30. doi:10.1016/S0023-6438(95)80008-5

[16] M. Oyaizu, "Studies on Product of Browning Reaction Prepared from Glucose Amine,” Japanese Journal of Nutrition, Vol. 44, No. 6, 1986, pp. 307-315. doi:10.5264/eiyogakuzashi.44.307

[17] F. N. Ko, G. Hsiao and Y. H. Kuo "Protection of Oxidative Hemolysis by Demethyldiisoeugenol in Normal and Beta-Thalassemic Red Blood Cells,” Free Radical Biology \& Medicine, Vol. 22, No. 1-2, 1997, pp. 215-222.

[18] E. Beutler, O. Duron and B. M. Kelly, "Improved Method for the Determination of Blood Glutathione," Journal of Laboratory and Clinical Medicine, Vol. 61, No. 3, 1963, pp. 882-888.

[19] J. T. Dodge, C. Mitchell and D. J. Hanahan, “The Preparation and Chemical Characteristics of Hemoglobin-Free Ghosts of Human Erythrocytes," Archives of Biochemistry and Biophysics, Vol. 100, No. 1, 1963, pp. 119-130. doi:10.1016/0003-9861(63)90042-0

[20] M. M. Bradford, "A Rapid and Sensitive Method for the Quantification of Microgram Quantities of Protein Utilizing the Principle of the Protein Dye Binding," Analytical Biochemistry, Vol. 72, No. 1-2, 1976, pp. 248-254. doi:10.1016/0003-2697(76)90527-3
[21] M. Skerget, P. Kotnik, M. Hadolin, A. R. Hras, M. Simonic and Z. Knez, "Phenols, Proanthocyanidins, Flavones and Flavonols in Some Plant Materials and Their Antioxidant Activities," Food Chemistry, Vol. 89, No. 2, 2005, pp. 191-198. doi:10.1016/j.foodchem.2004.02.025

[22] V. Saritha, K. R. Anilakumar and F. Khanum, "Antioxidant and Antibacterial Activity of Aloe vera Gel Extracts," International Journal of Pharmaceutical \& Biological Archive, Vol. 1, No 4, 2010, pp. 376-384.

[23] G. Williamson and C. Manach, "Bioavailability and Bioefficacy of Polyphenols in Humans. II. Review of 93 Intervention Studies," The American Journal of Clinical Nutrition, Vol. 81, No. 1, 2005, pp. 243S-255S.

[24] M. Miki, H. Tamai, M. Mino, Y. Yamamoto and E. Niki, "Free Radical Chain Oxidation of Rat Red Blood Cells by Molecular Oxygen and Its Inhibition by A-Tocopherol," Archives of Biochemistry and Biophysics, Vol. 258, No. 2, 1987, pp. 373-380. doi:10.1016/0003-9861

[25] L. L. Peters, R. A. Shivdasani, S.-C. Liu, M. Hanspal, K. M. John, J. M. Gonzalez, C. Brugnara, B. Gwynn, N. Mohandas, S. L. Alper, S. H. Orkin and S. E. Lux, “Anion Exchanger 1 (Band 3) Is Required to Prevent Erythrocyte Membrane Surface Loss but Not to Form the Membrane Skeleton,” Cell, Vol. 86, No. 6, 1996, PP. 917-927. doi:10.1016/S0092-8674(00)80167-1

[26] G. J. C. G. M. Bosman, M. Stappers and V. M. J. Novotný, "Changes in Band 3 Structure as Determinants of Erythrocyte Integrity during Storage and Survival after Transfusion,” Blood Transfusion, Vol. 8, No. 3, 2010, pp. s48-s52. doi:10.2450/2010.008S 